\title{
Assessment of Blade Mistuning Effects via Power Flow Analysis of Tuned Bladed Disks
}

\author{
Sanghum Baik, Matthew P. Castanier ${ }^{\dagger}$ and Christophe Pierre ${ }^{\ddagger}$ \\ Department of Mechanical Engineering,
}

The University of Michigan, Ann Arbor, MI 48109-2125, USA

\begin{abstract}
An efficient method is proposed for the early assessment of bladed disk designs with respect to blade mistuning effects. Structural and material differences among blades, known as blade mistuning, often cause significant increases in blade forced vibration amplitudes relative to ideal (tuned) bladed disks, and such effects are most pronounced when the blades and the disk share vibration energy actively. In this paper, it is shown that the flow of vibration energy between the blades and the disk of tuned bladed disks can be used to characterize disk-blade dynamic interaction, and consequently it is proposed as a means to assess blade mistuning effects. Power flow is first calculated for a simple lumped-parameter model, and it is shown to provide a physical explanation for the sharp increases in blade forced response amplitudes that take place when disk-blade dynamic interactions are strong. The power flow analysis is then extended to parametric design studies of finite element models for industrial bladed disks. The calculation of coupling power flow between the blade and the disk segment for a nominal bladed disk sector is shown to enable the rapid, albeit approximate, assessment of mistuning effects on blade vibration response for the nominal bladed disk design as well as for a variety of alternate designs obtained by varying disk structural parameters. The power flow analysis tool developed in this paper solely requires tuned system information, and thus it provides an inexpensive alternative to detailed statistical analyses of mistuned system response, such as Monte Carlo simulations. It seems particularly well suited to the design optimization of bladed disks with respect to minimizing blade mistuning effects.
\end{abstract}

\section{Introduction}

$\mathrm{T}$ HE bladed disk is a critical element in turbine jet engine rotors and turbomachinery in general. For these structural systems, blade-to-blade differences are unavoidable in practice, caused by manufacturing tolerances and in-service wear. Although such blade mistuning is typically small, it may result in considerable increases in forced vibration response blade amplitude and stress, thus causing fatigue, cracks, and possibly even blade failure. Accordingly, the topic of mistuning in bladed disks has attracted much attention, and literature surveys $s^{1 / 2}$ provide a thorough introduction to the physical phenomena caused by blade mistuning and to the computational methods developed to evaluate its effects on bladed disk free and forced responses.

The uncertain, random characteristics of blade mistuning require a statistical approach, and Monte Carlo simulations are typically carried out in order to assess the effects of blade mistuning on forced vibration response. However, a Monte Carlo simulation typically requires a large number of realizations of mistuned bladed disks, which carries a prohibitive computational cost for typical finite element models of industrial bladed disks. The high cost of Monte Carlo simulations can certainly be alleviated through the use of small-size reduced-order models. ${ }^{[3 / 5}$ However, such reduced-order models must be constructed from the finite element model at each iteration during an optimal design process, which both carries considerable computational cost and precludes automating the assessment of blade mistuning effects. Alternatively, there have been several interesting studies that investigate the asymptotic behaviors of

${ }^{*}$ Graduate Student Research Assistant, AIAA member

${ }^{\dagger}$ Associate Research Scientist, AIAA senior member.

${ }^{\ddagger}$ Stephen P. Timoshenko Collegiate Professor, AIAA senior member. 
the probabilistic dynamics of mistuned bladed disks. ${ }^{6718}$ However, these studies employed lumped-parameter models rather than finite element models. Thus, a practical, inexpensive tool is needed for the early assessment of blade mistuning effects in industrial bladed disks. This is the topic of this paper.

The characteristics of the natural frequency curves of tuned bladed disks, when plotted versus the number of nodal diameters of the corresponding mode shapes, play a key role in the sensitivity to mistuning. In particular, frequency curves that correspond to disk-dominated and blade-dominated families of modes may come close to one another and veer away abruptly from each other as the number of nodal diameters increases, a phenomenon known as "curve veering". For operating conditions in the veering regions, very large amplifications in blade vibration amplitudes due to mistuning have been observed, ${ }^{9}$ on the order of several hundred percent. Also importantly, several studies ${ }^{10 / 11 / 12}$ revealed that the curve veering phenomenon is closely related with the mechanisms of sharing vibration energy between the blades and the disk; therefore, strong disk-blade dynamic interactions typically result in significant blade mistuning effects.

In this paper, the above mechanism is utilized as a key argument to assess mistuning effects based on tuned system information only. Namely, a power flow analysis is proposed to characterize the dynamic interaction between the blades and the disk of a tuned bladed disk. This enables the computation of the magnitude and direction of the vibration energy propagation within the bladed disk, and thus the identification of the power transmission paths between the blades and the disk. The blade-to-disk coupling power is used to estimate mistuning effects on blade forced response, thus providing an indicator of mistuning sensitivity solely based on a tuned analysis.

The power flow analysis is first developed for a simple lumped-parameter model. This provides clear insight as to why a strong disk-blade interaction (which is usually, but not necessarily, observed in the veering regions) leads to large amplitude magnifications due to blade mistuning. Namely, it is shown that significant disk-blade dynamic coupling allows for a sufficient amount of vibration energy within the disk, and that the effect of blade mistuning is to reverse the energy flow from the disk to a few of the blades in the assembly, consequently causing a significant forced response increase for these blades.

Based upon the understanding of this basic mechanism, the power flow analysis is then extended to finite element representations of industrial bladed disks, by calculating the vibration energy flow between the blade and the disk segment for a nominal bladed disk sector. Again, the proposed power flow analysis is shown to predict the operating conditions that result in large amplifications in blade vibration amplitudes due to mistuning, based solely on tuned system calculations. In additions, for a variety of alternate bladed disk designs, the suggested analysis is also able to predict the correct trends in mistuning sensitivity.

The primary original contribution of this paper is the development of an efficient technique to evaluate blade mistuning effects based solely on tuned bladed disk analyses. The proposed power flow analysis is a fast and inexpensive alternative to costly Monte Carlo statistical analysis. It can be used to provide an estimate of mistuning effects early in the design process, and this preliminary assessment can then be used to make changes in the design of the bladed disk until a satisfactory sensitivity to mistuning is achieved. It is important to note that the new approach is not meant to be a substitute for Monte Carlo simulations, but rather to be complementary to it. In the design and analysis cycles, the rapid, albeit approximate, assessment of mistuning effects would be performed first, possibly leading to design changes. Once a design is finalized, comprehensive Monte Carlo simulations can be performed to predict accurately the statistics of the blade forced response amplitudes.

This paper is organized as follows. In section II, the vibration energy flow is formulated for a lumped-parameter bladed disk model with two degrees-of-freedom per sector. A representative bladed disk is then considered, and power flow analysis is utilized to elucidate the basic underlying mechanism that governs mistuning effects in veering regions with strong disk-blade dynamic interactions. Then, in section [III, the power flow analysis is extended to finite element models of bladed disks. Using three representative bladed disk models, it is shown that a power flow analysis of the tuned system can be used to estimate forced response sensitivity to mistuning for various design configurations. Finally, conclusions are provided. 


\section{Vibration Energy Flow in Bladed Disks}

The distribution of energy within a tuned bladed disk is investigated using a lumped-parameter model, and particular attention is paid to the "veering regions" of strong disk-blade interaction. This provides the basic mechanism from which to estimate blade mistuning effects based on tuned system information.

\section{A. Power Balance Formulation}

Figure 1 shows a lumped-parameter model of a bladed disk. The model features two degrees-of-freedom (DOF) for each sector, one for the blade and one for the disk segment. The blade mass is $m^{b}$ and the disk segment mass is $m^{d}$. Each sector $i$ features two shear-stiffness elements, $k_{i}^{b}$ and $k^{d}$. The blade mistuning is modeled as a variation in blade-alone stiffness, as

$$
k_{i}^{b}=k_{0}^{b}\left(1+\Delta k_{i}\right), \quad i=1,2, \ldots, N,
$$

where $N$ is the number of blades or sectors. The adjacent disk segments are connected with a single spring element of stiffness $k^{c}$. The external forcing is given as harmonic excitations of frequency $\omega$, and its amplitudes on each blades are given as $\mathbf{f}_{\mathrm{B}}$. When $\mathrm{x}$ denotes the steady-state response amplitudes for this excitation, the equations of motion for the lumped-parameter model are given as in the following way:

$$
\mathbf{M}=\left[\begin{array}{cc}
\operatorname{diag}\left(m^{d}\right) & \mathbf{0} \\
\mathbf{0} & \boldsymbol{d i a g}\left(m^{b}\right)
\end{array}\right], \quad \mathbf{K}=\left[\begin{array}{cc}
\mathbf{K}_{\mathrm{DD}} & -\mathbf{K}_{\mathrm{BB}} \\
-\mathbf{K}_{\mathrm{BB}} & \mathbf{K}_{\mathrm{BB}}
\end{array}\right], \quad \mathbf{x}=\left\{\begin{array}{c}
\mathbf{x}_{\mathrm{D}} \\
\mathbf{x}_{\mathrm{B}}
\end{array}\right\}, \quad \mathbf{f}=\left\{\begin{array}{c}
0 \\
\mathbf{f}_{\mathrm{B}}
\end{array}\right\},
$$

where $\gamma$ is the structural damping. The submatrices in the stiffness matrix are given as

$$
\begin{aligned}
& \mathbf{K}_{\mathrm{BB}}=k_{0}^{b} \cdot \operatorname{diag}\left(1+\Delta k_{i}\right), \\
& \mathbf{K}_{\mathrm{DD}}=\operatorname{circ}\left(2 k^{c}+k^{d},-k^{c}, \ldots,-k^{c}\right)+k_{0}^{b} \cdot \operatorname{diag}\left(1+\Delta k_{i}\right) .
\end{aligned}
$$

Here diag denotes a diagonal matrix whose elements are given in the argument, and circ denotes a circulant matrix whose first row is given in the argument.

The power flow of the lumped-parameter model is now formulated. The average power input to blade $i$ for a single period, $\Pi_{\mathrm{B}, i}^{i n}$, is computed as

$$
\Pi_{\mathrm{B}, i}^{i n}=\frac{1}{2} \operatorname{Re}\left[f_{\mathrm{B}, i}^{*} \cdot j \omega x_{\mathrm{B}, i}\right], \quad i=1,2, \ldots, N,
$$

where " $*$ " denotes a complex conjugate, and a positive value means that energy flows into the blade. The average power dissipated within a blade for a sigle period, $\Pi_{\mathrm{B}, i}^{d}$, is calculated in a similar manner as

$$
\begin{aligned}
\Pi_{\mathrm{B}, i}^{d} & =\frac{1}{2} \operatorname{Re}\left[-j \gamma k_{0}^{b}\left(1+\Delta k_{i}\right)\left(x_{\mathrm{B}, i}^{*}-x_{\mathrm{D}, i}^{*}\right) \cdot j \omega\left(x_{\mathrm{B}, i}-x_{\mathrm{D}, i}\right)\right] \\
& =\frac{1}{2} \omega \gamma k_{0}^{b}\left(1+\Delta k_{i}\right)\left|x_{\mathrm{B}, i}-x_{\mathrm{D}, i}\right|^{2}, \quad i=1,2, \ldots, N
\end{aligned}
$$

Next, the energy flow between the blades and the disk is calculated. Care must be taken to define the coupling power from the blades to the disk for the lumped-parameter model, since there is no explicit DOF associated with the 
blade-disk interface. Thus, a power balance relation is used, as follows. First, the complex conjugate of the external force on blade $i$ is expressed using Eq. (2) as

$$
f_{\mathrm{B}, i}^{*}=-\omega^{2} m^{b} x_{\mathrm{B}, i}^{*}+(1-j \gamma) k_{0}^{b}\left(1+\Delta k_{i}\right)\left(x_{\mathrm{B}, i}^{*}-x_{\mathrm{D}, i}^{*}\right), \quad i=1,2, \ldots, N .
$$

The power input to blade $i$ can then be expressed using Eq. (5), as follows:

$$
\frac{1}{2} \operatorname{Re}\left[f_{\mathrm{B}, i}^{*} \cdot j \omega x_{\mathrm{B}, i}\right]=\frac{1}{2} \operatorname{Re}\left[\left(f_{\mathrm{B}, i}^{*}+\omega^{2} m^{b} x_{\mathrm{B}, i}^{*}\right) \cdot j \omega x_{\mathrm{D}, i}\right]+\frac{1}{2} \omega \gamma k_{0}^{b}\left(1+\Delta k_{i}\right)\left|x_{\mathrm{B}, i}-x_{\mathrm{D}, i}\right|^{2},
$$

where $i=1,2, \ldots, N$. This power balance relation shows that the input power to a blade is split into power dissipated within the blade and power transmitted to the disk. The average blade-to-disk coupling power flow for a single period, $\Pi_{\mathrm{B}, i}^{c}$, at sector $i$ is now expressed as

$$
\Pi_{\mathrm{B}, i}^{c}=\frac{1}{2} \operatorname{Re}\left[\left(f_{\mathrm{B}, i}^{*}+\omega^{2} m^{b} x_{\mathrm{B}, i}^{*}\right) \cdot j \omega x_{\mathrm{D}, i}\right], \quad i=1,2, \ldots, N .
$$

A positive value indicates that power is transmitted from the blade to the disk.

The vibration power flow that propagates within the disk can be calculated in a similar manner. The blade-to-disk coupling power flow, which is the source of energy circulating within the disk, can be expressed using Eqs. (5) and (7) as follows:

$$
\Pi_{\mathrm{B}, i}^{c}=\frac{1}{2} \operatorname{Re}\left[(j \omega+\omega \gamma)\left\{-k^{c}\left(x_{\mathrm{D}, i-1}^{*}-x_{\mathrm{D}, i}^{*}\right) x_{\mathrm{D}, i}-k^{c}\left(x_{\mathrm{D}, i+1}^{*}-x_{\mathrm{D}, i}^{*}\right) x_{\mathrm{D}, i}\right\}\right]+\frac{1}{2} \omega \gamma k^{d}\left|x_{\mathrm{D}, i}\right|^{2},
$$

where $i=1,2, \ldots, N$. This expression can be further decomposed into several terms of power transmission and dissipation for the disk segment, as

$$
\begin{aligned}
\Pi_{\mathrm{B}, i}^{c}= & \left\{\frac{1}{2} \operatorname{Re}\left[-k^{c}\left(x_{\mathrm{D}, i-1}^{*}-x_{\mathrm{D}, i}^{*}\right) \cdot j \omega x_{\mathrm{D}, i}\right]+\frac{1}{2} \operatorname{Re}\left[-\omega \gamma k^{c}\left(x_{\mathrm{D}, i-1}^{*}-x_{\mathrm{D}, i}^{*}\right) x_{\mathrm{D}, i}\right]\right\} \\
& +\left\{\frac{1}{2} \operatorname{Re}\left[-k^{c}\left(x_{\mathrm{D}, i+1}^{*}-x_{\mathrm{D}, i}^{*}\right) \cdot j \omega x_{\mathrm{D}, i}\right]+\frac{1}{2} \operatorname{Re}\left[-\omega \gamma k^{c}\left(x_{\mathrm{D}, i+1}^{*}-x_{\mathrm{D}, i}^{*}\right) x_{\mathrm{D}, i}\right]\right\} \\
& +\left\{\frac{1}{2} \omega \gamma k^{d}\left|x_{\mathrm{D}, i}\right|^{2}\right\} \\
= & \left(\Pi_{\mathrm{L}, i}^{c}+\Pi_{\mathrm{L}, i}^{d}\right)+\left(\Pi_{\mathrm{R}, i}^{c}+\Pi_{\mathrm{R}, i}^{d}\right)+\Pi_{\mathrm{D}, i}^{d}, \quad i=1,2, \ldots, N,
\end{aligned}
$$

where $\Pi_{\mathrm{L}, i}^{c}$ and $\Pi_{\mathrm{R}, i}^{c}$ are the powers transmitted from sector $i$ to sectors $i-1$, and $i+1$, respectively, where a positive values means that energy flows from sector $i$. The terms $\Pi_{\mathrm{L}, i}^{d}$ and $\Pi_{\mathrm{R}, i}^{d}$ denote the power losses associated with the coupling of adjacent disk sectors. The last term $\Pi_{\mathrm{D}, i}^{d}$ is the power dissipated due to disk motion at sector $i$.

Finally, the vibration power flow for the bladed disk can be summarized as

$$
\begin{aligned}
\Pi_{\mathrm{B}, i}^{i n} & =\Pi_{\mathrm{B}, i}^{c}+\Pi_{\mathrm{B}, i}^{d} \\
& =\left[\left(\Pi_{\mathrm{L}, i}^{c}+\Pi_{\mathrm{L}, i}^{d}\right)+\left(\Pi_{\mathrm{R}, i}^{c}+\Pi_{\mathrm{R}, i}^{d}\right)+\Pi_{\mathrm{D}, i}^{d}\right]+\Pi_{\mathrm{B}, i}^{d}, \quad i=1,2, \ldots, N .
\end{aligned}
$$

Namely, the power input into the $i$ th blade due to external forcing, $\Pi_{\mathrm{B}, i}^{i n}$, is both dissipated by the blade motion $\left(\Pi_{\mathrm{B}, i}^{d}\right.$ ) and transmitted to the disk $\left(\Pi_{\mathrm{B}, i}^{c}\right)$. The $i$ th disk sector receives coupling power flow from the $i$ th blade, a portion of which is dissipated within the disk due to its motion $\left(\Pi_{\mathrm{D}, i}^{d}\right)$, and the remainder of which is transmitted and dissipated to the neighboring disk sectors, $\left(\Pi_{\mathrm{L}, i}^{c}+\Pi_{\mathrm{L}, i}^{d}\right)$ and $\left(\Pi_{\mathrm{R}, i}^{c}+\Pi_{\mathrm{R}, i}^{d}\right)$.

\section{B. Application to a Lumped-Parameter Model}

The above power flow formulation is used to investigate disk-blade dynamic interactions for a case study rotor. Power flow paths are determined, and used to explain the circumstances under which blade mistuning significantly increases forced response amplitudes for bladed disks. 
The lumped-parameter model for the case study rotor is shown in figure 1 . The bladed disk features 29 blades. When the disk and structural coupling parameters are normalized with relevant blade properties as

$$
\bar{m}=\frac{m^{d}}{m^{b}}, \quad \bar{k}_{c}=\frac{k^{c}}{k_{0}^{b}}, \quad \bar{k}_{d}=\frac{k^{d}}{k_{0}^{b}},
$$

its parameters are herein given as $\bar{m}=380.53, \bar{k}_{c}=$ $2231.77, \bar{k}_{d}=55.32$, and the structural damping is 0.006 . These parameters were obtained from the tuned natural frequency curve of an industrial compressor rotor stage, and interested readers are referred further to the extensive study ${ }^{12}$ on this topic. Figure 2 depicts the tuned free vibration natural frequencies versus the number of nodal diameters. The $\omega_{n}$ denotes the natural frequency of the structural system and $\omega_{0}^{b}$ denotes the nominal blade-alone natural frequency defined as $\left.\sqrt{(} k_{0}^{b} / m^{b}\right)$. As can be seen, this bladed disk features a frequency veering near the second nodal diameter; therefore, an external excitation of engine order 2 is chosen as the operating condition of interest. The engine order excitation denotes a forcing which is harmonic in time and forms a traveling wave along the circumference of the bladed disk with a constant phase angle between adjacent blades. An engine order 2 excitation for a 29 -blade rotor corresponds to a 24.83 degree interblade phase angle.

The power flow quantities are now computed for a blade of the tuned bladed disk subject to engine order 2 forcing, near the two resonant frequencies. The lower resonant frequency corresponds to a blade-dominated mode, and the higher one to a disk-dominated mode. For the blade-dominated mode resonance, figure 3 a) shows that the power input to the blade is primarily dissipated through the blade motion, whereas only a very small amount of power is transmitted to the disk. The opposite occurs for the disk-dominated mode resonance. Figure 3 (b) shows that most

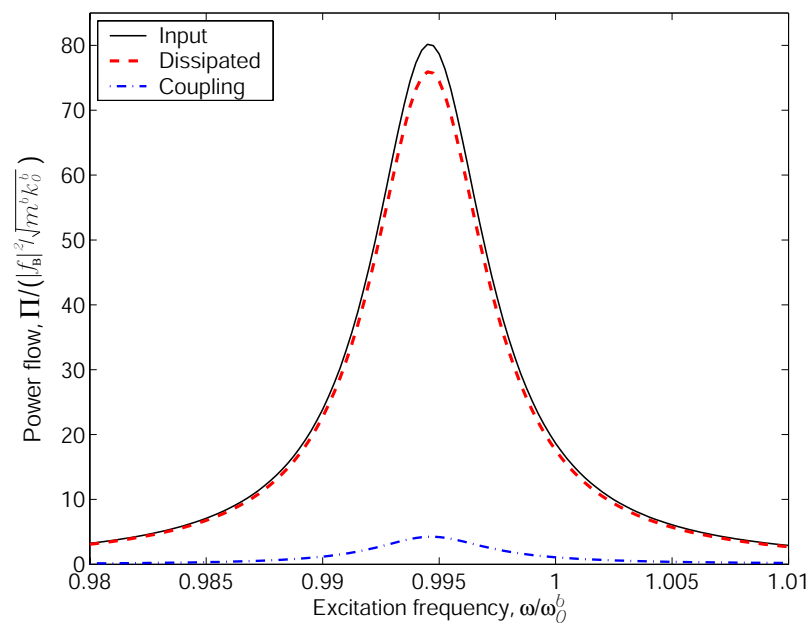

(a) Blade-dominated mode excitation

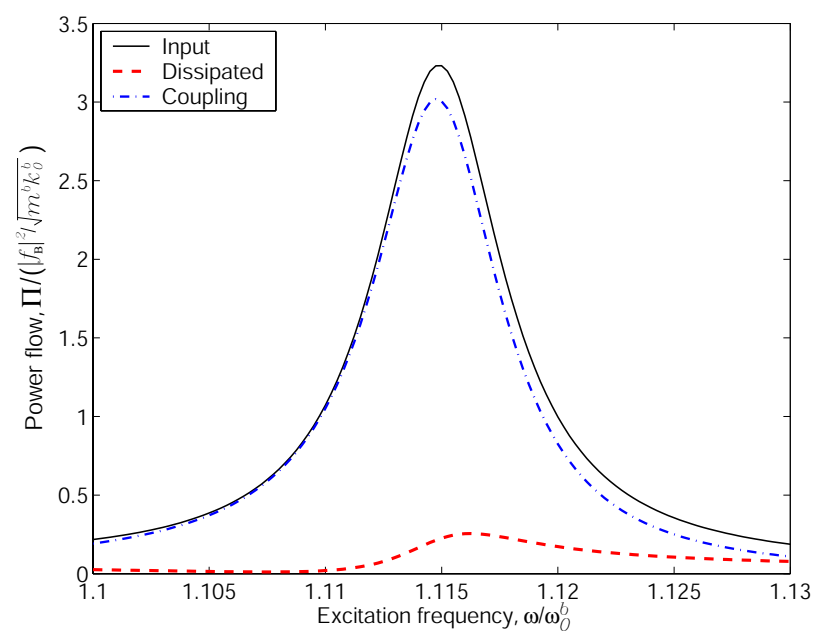

(b) Disk-dominated mode excitation

Figure 3. Vibration power flows for the tuned bladed disk lumped-parameter model, for engine order 2 excitation. 


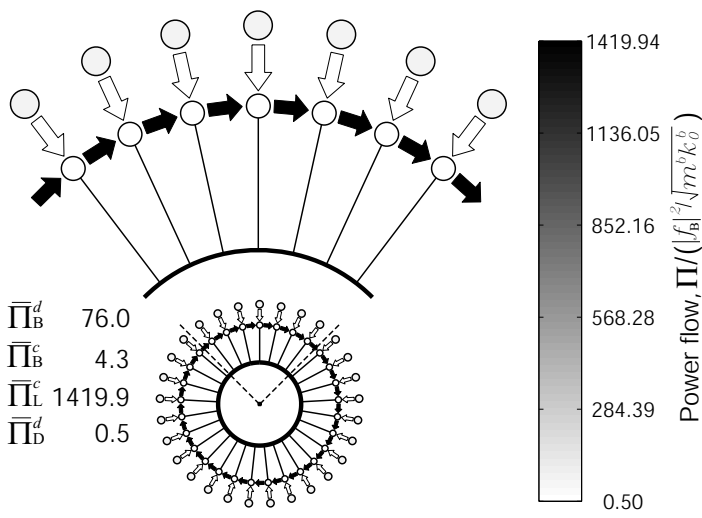

(a) Tuned bladed disk: EOX 2

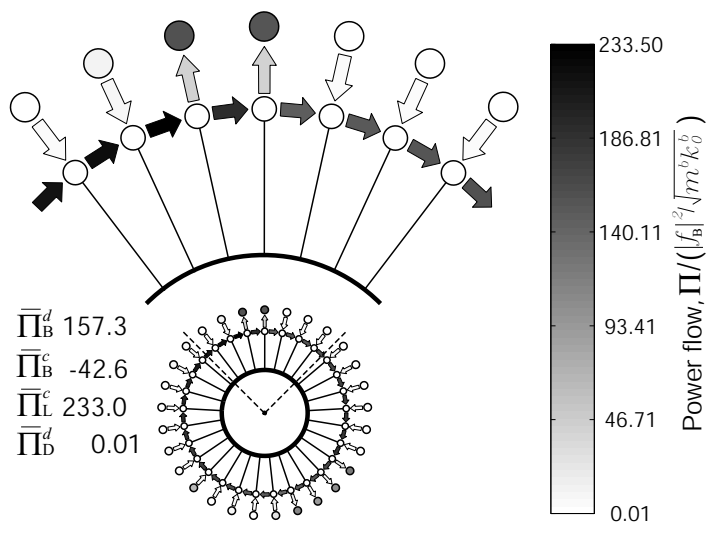

(c) Mistuned bladed disk: EOX 2

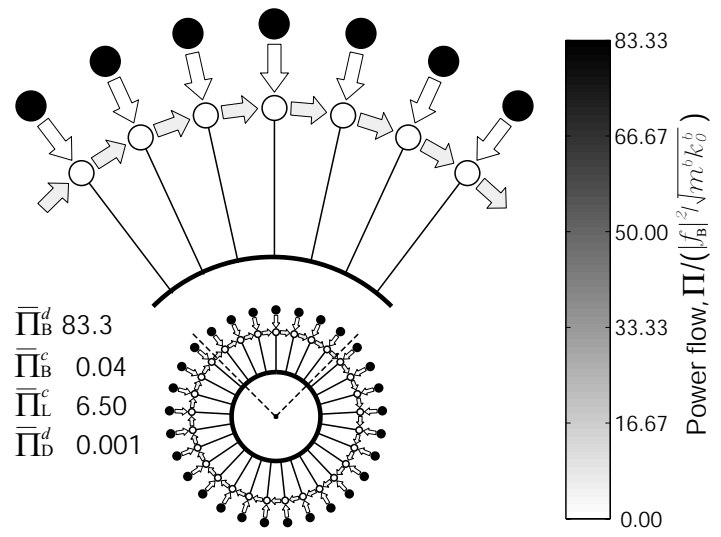

(b) Tuned bladed disk: EOX 5

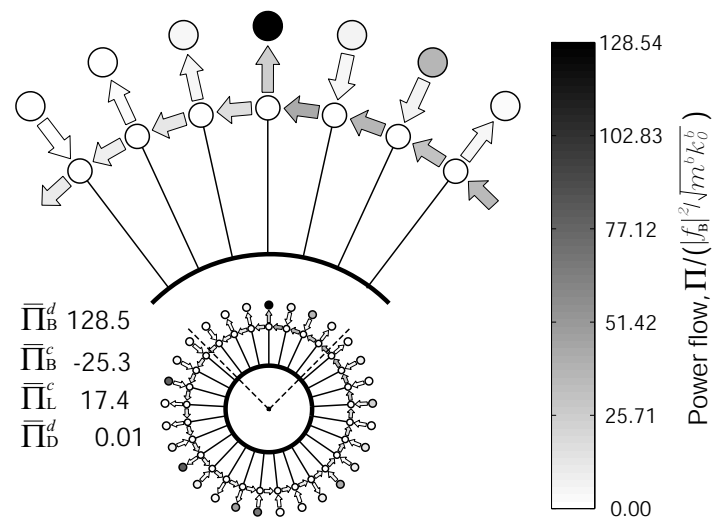

(d) Mistuned bladed disk: EOX 5

Figure 4. Vibration power flows for the tuned and mistuned bladed disks: (a) and (c) for the veering region excitation, and, (b) and (d) for the nonveering region excitation. In the mistuned cases, the power flow values are for the blade with the largest motion, and EOX denotes the order of the excitation.

of the power input is transmitted to the disk, as featured by the large values of the blade-to-disk coupling power flow throughout the resonant range. Therefore, the disk-blade dynamic interaction can be captured by computing what proportion of the power input to the blade is transmitted to the disk; herein, at the blade-dominated resonance only $5.28 \%$ of the power input is transferred to the disk, whereas for the disk-dominated mode excitation $90.61 \%$ is transmitted. Also, note the much smaller values of power flows for the disk-dominated resonance, in figure 3 (b), than for the blade-dominated one, as in figure 3 (a), due to the disk being much stiffer than the blade.

The above power flow analysis provides physical insight into how disk-blade dynamic interactions affect blade forced response, and in particular into the critical role they play regarding the sensitivity of blade response to mistuning. The case of a tuned bladed disk is first discussed. Engine order 2 and 5 excitations are considered, which correspond to excitations near veering and nonveering regions, respectively. Figure 4(a) depicts the power flows of the bladed disk, excited at resonant frequencies associated with the blade-dominated mode by the engine order 2 excitation that forms a traveling wave rotating in the clockwise direction. The "overbar" above each legend denotes that the power flow values are calculated being nondimensionalized with $\left|f_{B}\right|^{2} / \sqrt{m^{b} k_{0}^{b}}$. It shows that for the veering excitation, relatively large amounts of vibration energy are transmitted from the blade to the disk, as featured by the blade-to-disk coupling power flow of 4.3, providing considerable vibration energy of 1419.9 that circulates within the 
Table 1. Mistuning pattern estimated from blade-alone eigenvalue measurements of a prototype bladed disk. The mistuning pattern, shown herein, is calculated as $\left(\left(\omega_{\mathrm{mt}}^{b} / \omega_{0}^{b}\right)^{2}-1\right) \times 100$, where $\omega_{\mathrm{mt}}^{b}$ denotes the blade-alone natural frequency of the mistuned structural system.

\begin{tabular}{cc|cc|cc|cc} 
Blade & Mistuning, $\%$ & Blade & Mistuning, $\%$ & Blade & Mistuning, $\%$ & Blade & Mistuning, $\%$ \\
\hline 1 & 5.704 & 9 & 0.242 & 17 & 4.479 & 25 & -3.631 \\
2 & 1.207 & 10 & -2.747 & 18 & 3.030 & 26 & -1.665 \\
3 & 4.670 & 11 & -3.631 & 19 & 0.242 & 27 & 0.783 \\
4 & -1.502 & 12 & -3.570 & 20 & 1.734 & 28 & -1.169 \\
5 & 5.969 & 13 & -3.631 & 21 & 2.919 & 29 & -1.332 \\
6 & -3.324 & 14 & -3.631 & 22 & -0.328 & & \\
7 & -0.078 & 15 & 0.242 & 23 & 0.086 & & \\
8 & -1.688 & 16 & 4.934 & 24 & -3.654 & &
\end{tabular}

disk. This indicates that the blades and disk motions are strongly coupled. In turn, for the veering excitation, the blade forced responses become relatively small, as captured by the dissipated power within a blade of 76.0, because of the large energy transfer from the blades to the disk. However, this is not in the case of the nonveering excitation shown in figure 4(b); for instances, the blade-to-disk coupling power flow is very small as 0.04 , and the power circulating within the disk is just 6.50 .

For a mistuned bladed disk, the blade mistuning disrupts the transmission of vibration energy within the disk. Table 1 shows an example pattern of percentage variations in blade-alone eigenvalues for the mistuned bladed disk. For this system, as seen by the negative numbers (-42.6) for the blade-to-disk coupling power flow in figure 4 (c), the effect of blade mistuning is to reverse vibration power flow from the disk to a few of the blades, thus providing a large amount of vibration energy to these blades. The power dissipated within the blade of 157.3 is then much larger than in the tuned case, and also larger than the power input from external excitation. Consequently, due to significant disk-blade dynamic interactions, the bladed disk experiences significant increases, such as 2.07 times tuned vibration amplitude, of some blades due to mistuning. For the nonveering excitation of figure 4(d), the small disk-blade interactions observed in this case prevent vibration energy from propagating within the disk, and thus the blade vibration

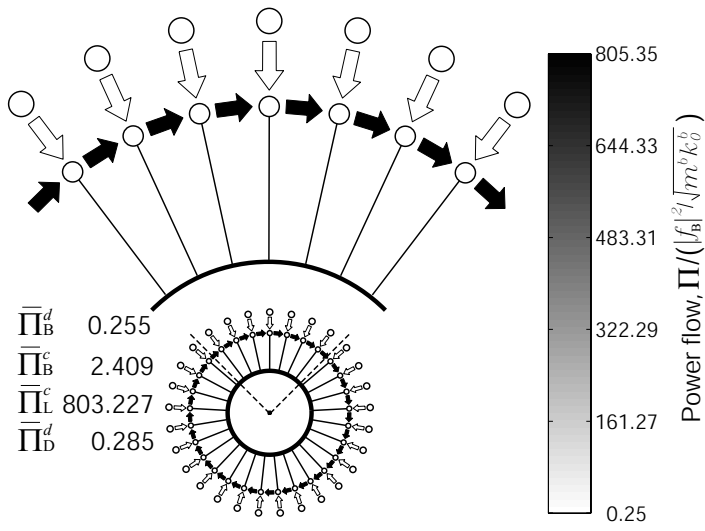

(a) Tuned bladed disk: EOX 2

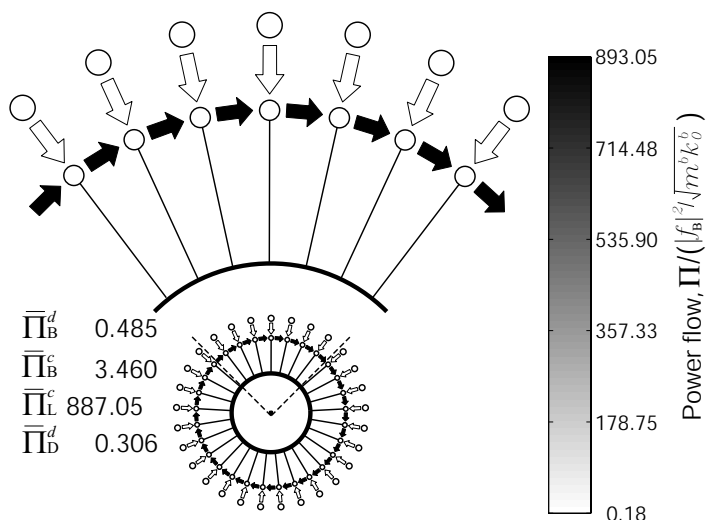

(b) Mistuned bladed disk: EOX 2

Figure 5. Vibration power flows for the tuned and mistuned bladed disks at resonant frequencies near to the disk-dominated mode. The engine order 2 excitation is used. 
amplifications due to mistuning become less significant: namely, 1.54 times tuned blade vibration amplitudes.

Meanwhile, strong disk-blade dynamic interactions typically result in negligible blade mistuning effects. Figures 5(a) and (b) depict the power flows of tuned and mistuned bladed disks at resonant frequencies near diskdominated modes for the engine order 2 excitation. As seen, for the tuned case, the strong coupling between the blades and disk causes most of the energy to be transmitted to the disk, thus causing negligible blade motions as captured by the dissipated power within a blade of 0.255 . When blade mistuning is present, the disk-dominated motion still prevails. Consequently, the blade vibration response is not localized, and no reversal of the coupling power flow from the disk to the blades is observed, causing very negligible blade vibration. Note that this matches with previous study results reported in the literature. $\frac{.1213}{113}$

In summary, power flow analysis can be used to capture disk-blade dynamic interactions. These can possibly serve as a key indicator for predicting the amplification of blade response amplitude due to mistuning. This key idea is explored further in the next sections, to predict mistuning sensitivity for industrial bladed disk models based solely on a tuned system power flow analysis.

\section{Prediction of Mistuning Sensitivity}

In this section, the power flow analysis is first extended to finite element models of bladed disks. The power flow balance for a single tuned sector is then utilized to assess mistuning sensitivity, and parametric design studies of representative and industrial bladed disks are carried out. The numerical experiments presented demonstrate that power flow analysis can be a useful tool for quickly evaluating bladed disk design with respect to blade mistuning effects.

\section{A. Power Flow Balance Formulation for Finite Element Models}

The input, coupling, and dissipated powers for a blade, introduced in section II for the lumped-parameter model, are extended to finite element models of bladed disks. In addition, the power dissipated within the blade is used to efficiently estimate the blade stress level.

For a bladed disk finite element model, the displacement amplitudes $\mathrm{x}$ of steady-state vibration responses can be expressed as

$$
\mathbf{x}=\left\{\begin{array}{c}
\mathbf{x}_{1} \\
\mathbf{x}_{2} \\
\vdots \\
\mathbf{x}_{N}
\end{array}\right\}, \quad \text { where } \quad \mathbf{x}_{i}=\left\{\begin{array}{c}
\mathbf{x}_{\mathrm{D}, i} \\
\mathbf{x}_{\Gamma, i} \\
\mathbf{x}_{\mathrm{B}, i}
\end{array}\right\}, \quad i=1,2, \ldots, N
$$

where the superscripts D, $\Gamma$, and B denote quantities associated with the disk interior, the disk-blade boundary, and the blade interior, respectively. For each blade-disk sector, the equations of motion are:

$$
\begin{aligned}
& \left(-\omega^{2}\left[\begin{array}{cc}
\tilde{\mathbf{M}}_{\mathrm{DD}, i} & \tilde{\mathbf{M}}_{\Gamma \mathrm{D}, i} \\
\tilde{\mathbf{M}}_{\mathrm{D} \Gamma, i} & \tilde{\mathbf{M}}_{\Gamma \Gamma, i}^{\mathrm{D}}
\end{array}\right]+(1+j \gamma)\left[\begin{array}{cc}
\tilde{\mathbf{K}}_{\mathrm{DD}, i} & \tilde{\mathbf{K}}_{\Gamma \mathrm{D}, i} \\
\tilde{\mathbf{K}}_{\mathrm{D} \Gamma, i} & \tilde{\mathbf{K}}_{\Gamma \Gamma, i}^{\mathrm{D}}
\end{array}\right]\right)\left\{\begin{array}{c}
\mathbf{x}_{\mathrm{D}, i} \\
\mathbf{x}_{\Gamma, i},
\end{array}\right\}=\left\{\begin{array}{c}
\mathbf{t}_{\mathrm{D}, i} \\
-\mathbf{t}_{\Gamma, i}
\end{array}\right\}, \\
& \left(-\omega^{2}\left[\begin{array}{cc}
\tilde{\mathbf{M}}_{\Gamma \Gamma, i}^{\mathrm{B}} & \tilde{\mathbf{M}}_{\mathrm{B} \Gamma, i} \\
\tilde{\mathbf{M}}_{\Gamma \mathrm{B}, i} & \tilde{\mathbf{M}}_{\mathrm{BB}, i}
\end{array}\right]+(1+j \gamma)\left[\begin{array}{cc}
\tilde{\mathbf{K}}_{\Gamma \Gamma, i}^{\mathrm{B}} & \tilde{\mathbf{K}}_{\mathrm{B} \Gamma, i} \\
\tilde{\mathbf{K}}_{\Gamma \mathrm{B}, i} & \tilde{\mathbf{K}}_{\mathrm{BB}, i}
\end{array}\right]\right)\left\{\begin{array}{l}
\mathbf{x}_{\Gamma, i} \\
\mathbf{x}_{\mathrm{B}, i}
\end{array}\right\}=\left\{\begin{array}{c}
\mathbf{t}_{\Gamma, i} \\
\mathbf{f}_{\mathrm{B}, i}
\end{array}\right\},
\end{aligned}
$$

where $\tilde{\mathbf{M}}$ and $\tilde{\mathbf{K}}$ are the mass and stiffness matrices, $\mathbf{t}_{\mathrm{D}, i}$ and $\mathbf{t}_{\Gamma, i}$ are the traction forces at the sector-to-sector boundary and the disk-blade boundary for sector $i$, respectively, and $\mathbf{f}_{\mathrm{B}, i}$ is the external forcing on blade $i$. The average input power to blade $i$ for a single period is given by:

$$
\Pi_{\mathrm{B}, i}^{i n}=-\frac{1}{2} \operatorname{Re}\left[j \omega \mathbf{x}_{\mathrm{B}, i}^{*, \mathrm{~T}} \cdot \mathbf{f}_{\mathrm{B}, i}\right], \quad i=1,2, \ldots, N .
$$


A positive value means that energy flows into the blade. The blade-to-disk coupling power flow can be computed in a similar manner. In doing so, the traction force at the disk-blade boundary DOF must first be computed in the following way:

$$
\mathbf{t}_{\Gamma, i}=-\omega^{2}\left(\tilde{\mathbf{M}}_{\Gamma \Gamma}^{\mathrm{B}} \mathbf{x}_{\Gamma, i}+\tilde{\mathbf{M}}_{\mathrm{B} \Gamma} \mathbf{x}_{\mathrm{B}, i}\right)+(1+j \gamma)\left(\tilde{\mathbf{K}}_{\Gamma \Gamma}^{\mathrm{B}} \mathbf{x}_{\Gamma, i}+\tilde{\mathbf{K}}_{\mathrm{B} \Gamma} \mathbf{x}_{\mathrm{B}, i}\right), \quad i=1,2, \ldots, N .
$$

Then, the average power from the blade to the disk at sector $i$ for a single period is given by:

$$
\Pi_{\mathrm{B}, i}^{c}=\frac{1}{2} \operatorname{Re}\left[j \omega \mathbf{x}_{\Gamma, i}^{*, \mathrm{~T}} \cdot \mathbf{t}_{\Gamma, i}\right], \quad i=1,2, \ldots, N
$$

A positive value indicates that energy is transmitted from the blade to the disk. Finally, the average dissipated power for a single period can be calculated as such:

$$
\Pi_{\mathrm{B}, i}^{d}=\Pi_{\mathrm{B}, i}^{i n}-\Pi_{\mathrm{B}, i}^{c}
$$

In summary, once blade forced responses are computed for the excitation considered, power flow for the bladed disk can be analyzed using Eqs. (15) to (18).

Moreover, the power flow analysis allows for the systematic estimation of disk-blade dynamic interaction within a single sector. The tuned Coupling Power Indicator (tCPI) is hereby defined as

$$
\mathrm{tCPI}=\sqrt{\Pi_{\mathrm{B}}^{c, 0} / \Pi_{\mathrm{B}}^{i n, 0}}
$$

where the superscript " 0 " denotes that power flow is calculated for a tuned bladed disk. In the next sections, this quantity will be used as an indicator to predict the blade vibration amplification due to mistuning, and a detailed validation of this suggested metric will be presented.

Next, the power dissipated within the blade is proposed as an index for estimating the blade stress amplification due to blade mistuning. The blade stress level is important in evaluating failure criteria of a bladed disk design. Thus, in practice, after blade response amplitudes have been calculated (typically using a reduced-order model), an additional finite element analysis is needed to compute the blade stress level. This procedure may carry a prohibitive cost for the statistical analysis of mistuned bladed disks, especially when an optimum is sought among a large number of design configurations. Therefore, in this paper, a metric is provided for estimating the blade stress level. The indicator is computed directly from blade displacements and eliminates the need for an additional finite element analysis.

In order to formulate this stress indicator, the power flow analysis for a sector is revisited. From Eqs. (16) and (17), the blade-to-disk coupling power flow is expressed as:

$$
\Pi_{\mathrm{B}, i}^{c}=-\frac{1}{2} \operatorname{Re}\left[j \omega \mathbf{x}_{\mathrm{B}, i}^{*, \mathrm{~T}} \cdot \mathbf{f}_{\mathrm{B}, i}\right]+\frac{1}{2} \operatorname{Re}\left[\pi_{\mathrm{B}, i}^{d}\right]=\Pi_{\mathrm{B}, i}^{i n}+\frac{1}{2} \operatorname{Re}\left[\pi_{\mathrm{B}, i}^{d}\right], \quad i=1,2, \ldots, N,
$$

where the complex quantity $\pi_{\mathrm{B}, i}^{d}$ is given as

$$
\begin{aligned}
\pi_{\mathrm{B}, i}^{d}=j \omega\{- & \omega^{2}\left(\mathbf{x}_{\mathrm{B}, i}^{*, \mathrm{~T}} \tilde{\mathbf{M}}_{\mathrm{BB}} \mathbf{x}_{\mathrm{B}, i}+\mathbf{x}_{\Gamma, i}^{*, \mathrm{~T}} \tilde{\mathbf{M}}_{\Gamma \Gamma} \mathbf{x}_{\Gamma, i}+2 \operatorname{Re}\left[\mathbf{x}_{\mathrm{B}, i}^{*, \mathrm{~T}} \tilde{\mathbf{M}}_{\Gamma \mathrm{B}} \mathbf{x}_{\Gamma, i}\right]\right) \\
& \left.+(1+j \gamma)\left(\mathbf{x}_{\mathrm{B}, i}^{*, \mathrm{~T}} \tilde{\mathbf{K}}_{\mathrm{BB}} \mathbf{x}_{\mathrm{B}, i}+\mathbf{x}_{\Gamma, i}^{*, \mathrm{~T}} \tilde{\mathbf{K}}_{\Gamma \Gamma} \mathbf{x}_{\Gamma, i}+2 \operatorname{Re}\left[\mathbf{x}_{\mathrm{B}, i}^{*, \mathrm{~T}} \tilde{\mathbf{K}}_{\Gamma \mathrm{B}} \mathbf{x}_{\Gamma, i}\right]\right)\right\} .
\end{aligned}
$$

The dissipated power can then be expressed using Eqs. (18) and (20) as follows:

$$
\Pi_{\mathrm{B}, i}^{d}=-\frac{1}{2} \operatorname{Re}\left[\pi_{\mathrm{B}, i}^{d}\right]=\omega \gamma\left(\frac{1}{2} \mathbf{x}_{\mathrm{B}, i}^{*, \mathrm{~T}} \tilde{\mathbf{K}}_{\mathrm{BB}} \mathbf{x}_{\mathrm{B}, i}+\frac{1}{2} \mathbf{x}_{\Gamma, i}^{*, \mathrm{~T}} \tilde{\mathbf{K}}_{\Gamma \Gamma} \mathbf{x}_{\Gamma, i}+\operatorname{Re}\left[\mathbf{x}_{\mathrm{B}, i}^{*, \mathrm{~T}} \tilde{\mathbf{K}}_{\Gamma \mathrm{B}} \mathbf{x}_{\Gamma, i}\right]\right), \quad i=1,2, \ldots, N
$$

For an isotropic elastic material, the term in parenthesis is the strain energy due to blade motion, $U_{\mathrm{B}, i}$. The dissipated power can thus be expressed as

$$
\Pi_{\mathrm{B}, i}^{d}=\omega \gamma U_{\mathrm{B}, i}, \quad i=1,2, \ldots, N .
$$


This suggests that the dissipated power in the blade, directly computed from blade displacements, can capture variations in stress. Consequently, the Stress Amplification Indicator (SAI) is defined as

$$
\mathrm{SAI}=\sqrt{\Pi_{\mathrm{B}}^{d, \mathrm{mt}} / \Pi_{\mathrm{B}}^{d, 0}}
$$

where the superscript "mt" is for a mistuned bladed disk.

In the next three sections, the power flow analysis developed herein is applied to three representative bladed disks. It is shown that a tuned sector analysis is able to predict blade stress amplification due to mistuning, both for the nominal designs and a variety of alternate designs obtained by varying disk parameters.

\section{B. Application to a Case Study Bladed Disk}

To validate the proposed power flow analysis, a simple bladed disk, referred to as the academic bladed disk, is used. Its finite element model is shown in figure 6(a), and a detailed description can be found in Ref. 14. It features total 24 blades, and, the material properties for the nominal design configuration are given in table $2 \mid$ The free vibration natural frequencies of the tuned system are plotted with respect to the number of nodal diameters in figure 6(b).

First, the SAI metric given in Eq. (23) is validated. To achieve this, the structural properties of the disk segment are varied by changing the Young's modulus and the mass density for the disk from $90 \%$ to $110 \%$ of their nominal values. Then, for each configuration, the bladed disk is mistuned using the pattern of blade-alone eigenvalue variations listed in table 3. The SAI values are then calculated for each blade at the resonant frequencies, using an efficient reduced-order

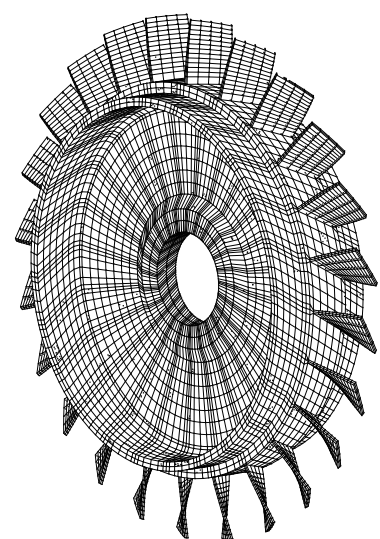

(a)

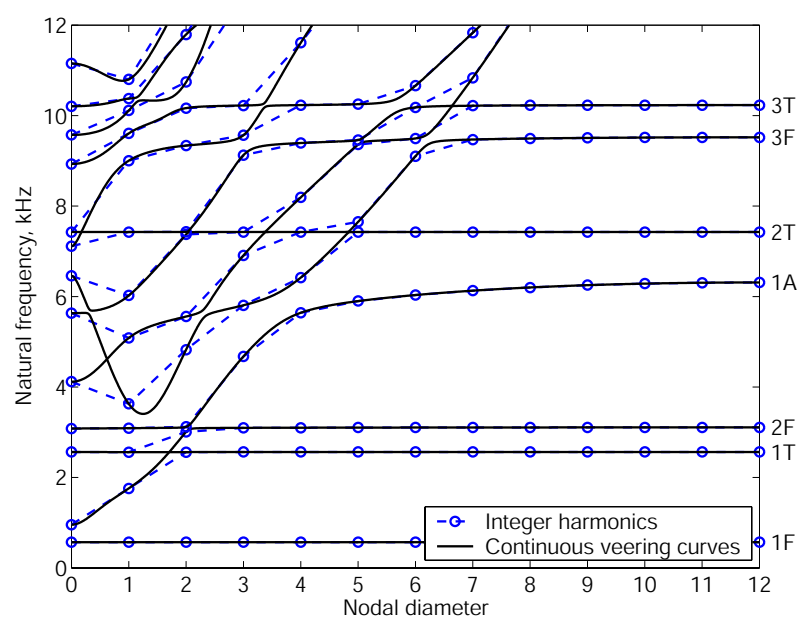

(b)

Figure 6. The academic bladed disk assembly: (a) finite element model, and (b) tuned natural frequency curves.

Table 2. Material properties of the academic bladed disk.

\begin{tabular}{lcc} 
Material property & Value & Unit \\
\hline Modulus of Elasticity & 200.0 & $\mathrm{GPa}$ \\
Mass Density & 7860 & $\mathrm{~kg} / \mathrm{m}^{3}$ \\
Structural damping & 0.0025 & -
\end{tabular}

10 of 20 
Table 3. Variations in blade-alone eigenvalue for the mistuned academic bladed disk. The mistuning pattern, shown herein, is calculated as $\left(\left(\omega_{\mathrm{mt}}^{b} / \omega_{0}^{b}\right)^{2}-1\right) \times 100$, where $\omega_{\mathrm{mt}}^{b}$ denotes the blade-alone natural frequency of the mistuned academic bladed disk.

\begin{tabular}{cc|cc|cc} 
Blade & Mistuning, $\%$ & Blade & Mistuning, $\%$ & Blade & Mistuning, $\%$ \\
\hline 1 & -0.515 & 9 & 2.930 & 17 & -0.415 \\
2 & -1.875 & 10 & 2.720 & 18 & 0.430 \\
3 & -1.820 & 11 & 2.770 & 19 & 1.840 \\
4 & -0.390 & 12 & -4.925 & 20 & -5.475 \\
5 & -5.005 & 13 & -8.075 & 21 & 2.395 \\
6 & -0.850 & 14 & -4.905 & 22 & 3.810 \\
7 & 1.405 & 15 & 5.935 & 23 & 4.110 \\
8 & 7.620 & 16 & -6.925 & 24 & 3.930
\end{tabular}

mode $[\sqrt{5}$ for the blisk. Similarly, the amplification in blade stress due to blade mistuning is computed, also at the resonant frequencies, using a direct finite element analysis. An engine order 2 excitation of the $2 \mathrm{~F}$ blade mode family is considered, and the nodes at the blade tips are excited by applying a combination of axial and circumferential forces with unit amplitudes and a 30-degree phase delay. These two metrics are plotted against each other in figure 7. Observe that the SAI is able to capture reasonably well the blade stress amplification due to mistuning for all disk configurations considered, albeit at a negligible cost compared to a finite element analysis. Thus in the rest of the paper, the SAI, which is directly obtained from the blade response amplitudes using a power flow analysis, will be used as the indicator for blade stress amplification due to blade mistuning.

Returning to the academic blisk with nominal disk parameters, power flow analysis is applied to determine the operating conditions that are most sensitive to blade mistuning. Figure 8 a) depicts the tCPI values, obtained

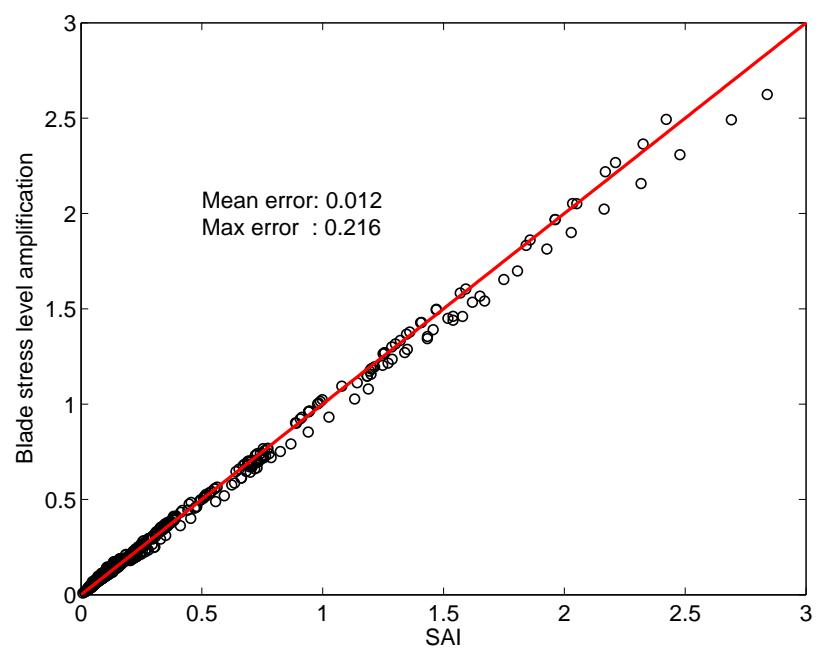

Figure 7. Correlation of the SAI with the amplification in blade stress level, for the academic bladed disk with various design configurations. The error values denote maximum and average absolute errors. using a single tuned sector, for a range of engine order excitations and three families of blade modes: 1T, 2F, and 2T. The power flow analysis indicates that the disk-blade dynamic interaction is most active for an engine order 2 excitation of the $2 \mathrm{~F}$ blade mode family. This would suggest that blade mistuning effects are most critical for this operating condition. In order to verify this conjecture, Monte Carlo simulations of the blade stress amplification metric, the SAI, are performed for these operating conditions. The largest values obtained for the 95th percentile SAI turn out to be 1.1340, 2.5203, 1.6241 for the $1 \mathrm{~T}, 2 \mathrm{~F}$, and $2 \mathrm{~T}$ excitations, respectively. Figure 8 (b) shows detailed results for the 2F-blade mode family excitation. The mistuning strength is the standard deviation value of a set of mistuning pattern used for the Monte Carlo simulation. Observe that the tuned Coupling Power Indicator clearly and correctly predicts the operating condition that is subject to the largest effects of blade mistuning.

Power flow analysis is now employed for predicting variations in mistuning sensitivity caused by disk design changes. Similarly to the previous numerical experiment, the disk structural parameters, such as stiffness and mass density, are perturbed from their nominal values. Then, for each design configuration, Monte Carlo simulations are performed to compute the 95th percentile SAI. Also, a power flow balance is performed for the tuned bladed disks to 


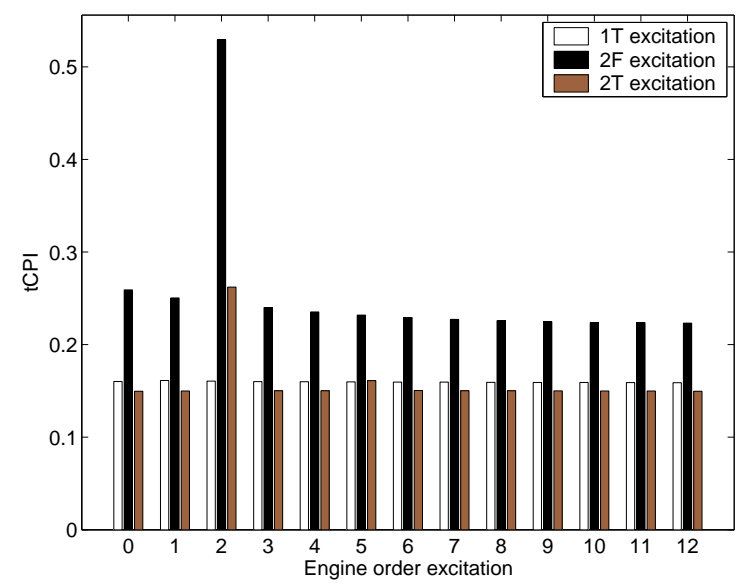

(a) Power flow analysis

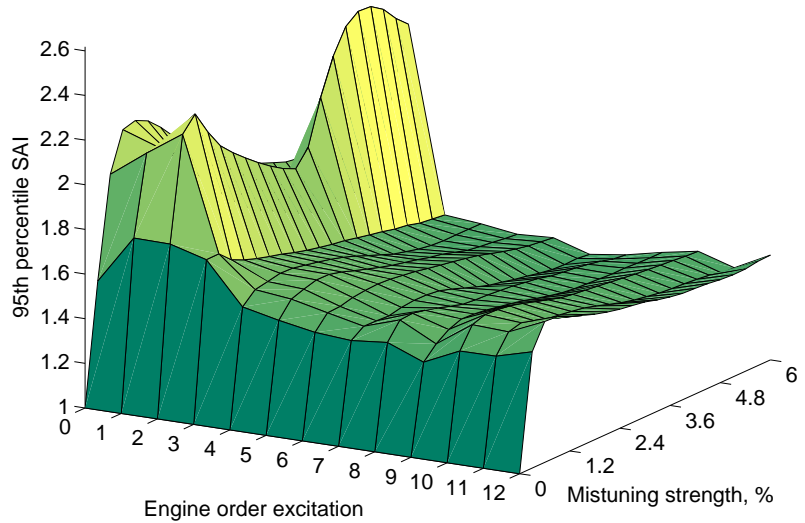

(b) Monte Carlo simulation for $2 \mathrm{~F}$-mode excitation

Figure 8. Prediction of blade mistuning effects for the academic bladed disk with nominal design configuration.

compute the tCPI. An engine order 2 excitation of the $2 \mathrm{~F}$ blade mode family is used as the operating condition, based on the result in the nominal design case.

As can be seen in figures 9 a) and (b), the power flow analysis predicts not only the correct trends, but also which of the design configurations results in the most significant blade mistuning effects, for both structural parameter changes. These data are plotted against each other in figure 10 to further illustrate the strong correlation between them. The legend, $0.90 \mathrm{~m}_{0}$, denotes that the tCPI and SAI data are computed for an academic bladed disk of which disk mass density is featured as $90 \%$ of its nominal value. In a similar manner, the legend, $1.10 \mathrm{E}_{0}$, means that the data is from an academic bladed disk of which disk Young's modulus is changed to $110 \%$ of its nominal value, and so on. As

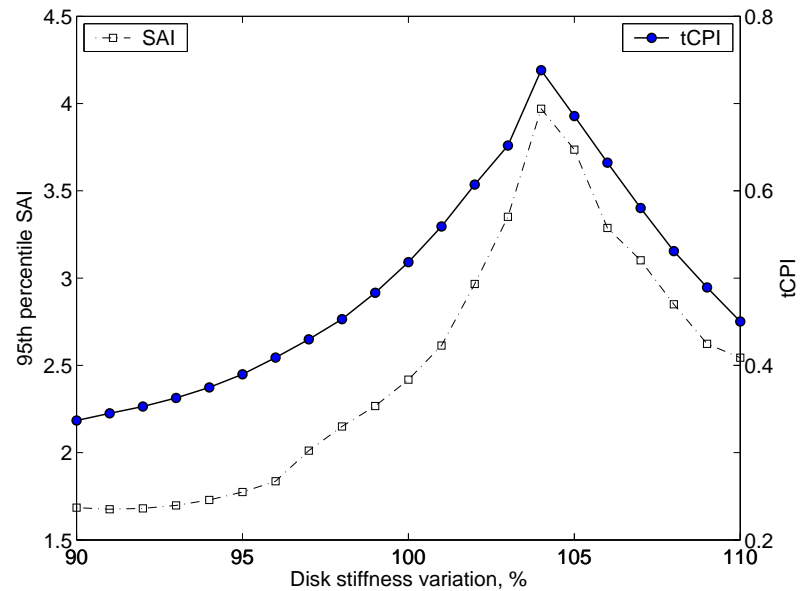

(a) Disk stiffness variation

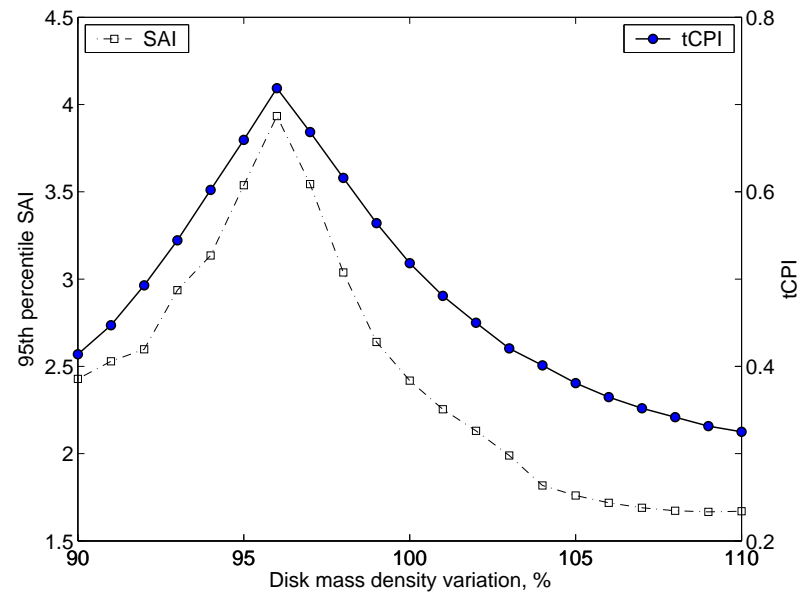

(b) Disk mass density variation

Figure 9. Predictions of mistuning sensitivity variations caused by changes in disk parameters, for the academic bladed disk. 
seen, there are actually two trends for each structural parameter variation: one tracking from a $90 \%$ variation to the parameter yielding the peak response, and the other tracking from this to the $110 \%$ parameter variation. Here $r^{2}$ is the correlation factor, calculated by the linear regression analysis of the plotted data. It shows that monotonic variations of blade vibration amplification can be predicted by the power flow analysis of the tuned bladed disk with $95 \%$ accuracy.

\section{Case Study: Industrial Compressor Rotor}

Based on the preliminary validation for the academic blisk, an industrial compressor rotor is now studied to further assess the ability of the power flow analysis to predict mistuning effects. Moreover, realistic design changes in disk geometry are considered, in addition to varying disk structural parameters. This may simulate a practical bladed disk design procedure.

Figure 11 (a) shows the finite element model of an industrial compressor rotor with 29 blades; see Ref. 4 for a detailed description. For the nominal design, the Young's

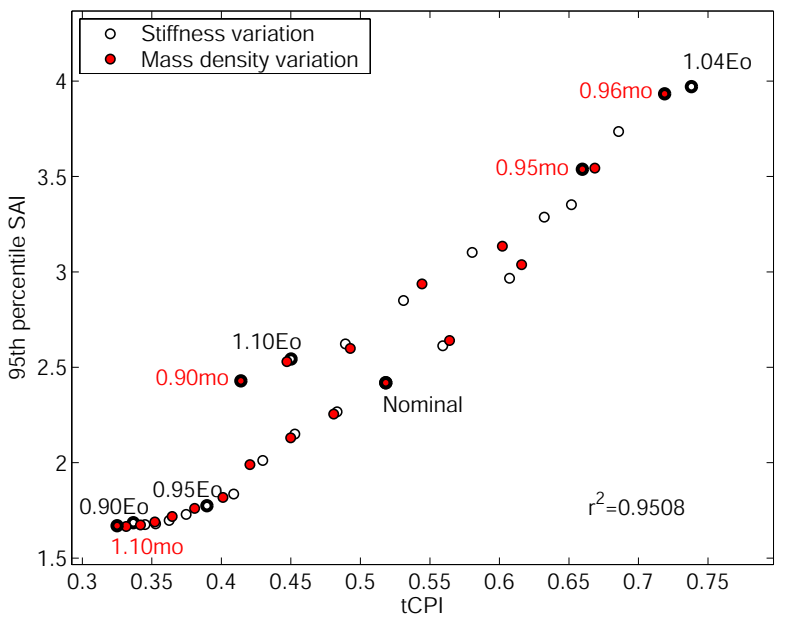
modulus is $203.3 \mathrm{GPa}$; the modulus of rigidity is 77.0 $\mathrm{GPa}$; the mass density is $7909 \mathrm{~kg} / \mathrm{m}^{3}$; the Poisson's ratio is 0.305 . Also, the structural damping value is given as 0.006 . For this nominal tuned rotor, the free vibration natural frequencies are plotted versus the number of nodal diameters in figure 11(b).

For the tuned bladed disk with nominal design configuration, the power flow analysis is at first employed to identify which operating conditions correspond to significant mistuning effects. For several frequency ranges, the nodes at the blade tips are excited with unit magnitude external forcing, normally to the blade surface. The tCPI values are then calculated at resonant frequencies for many engine order excitations on each blade mode family, and results are

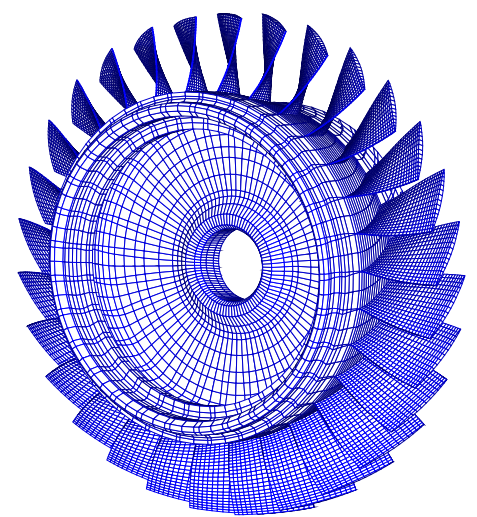

(a)

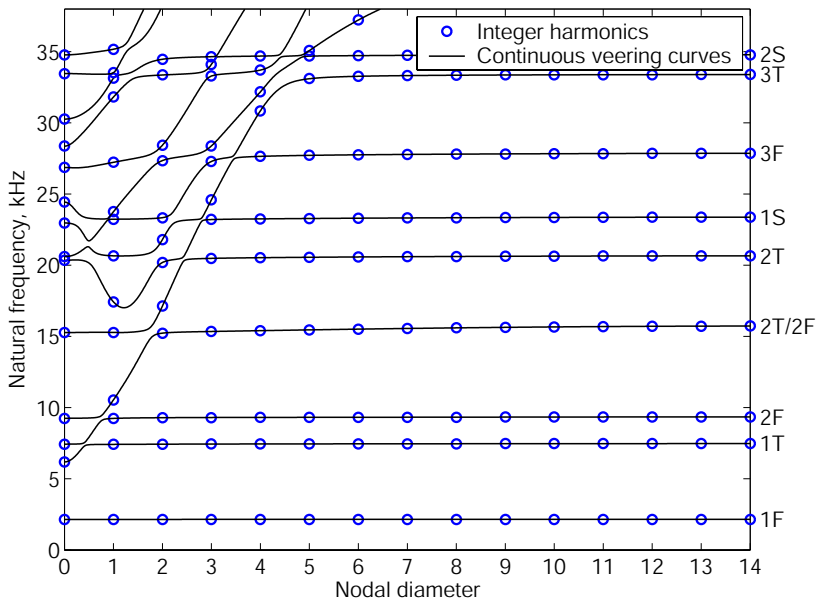

(b)

Figure 11. Industrial compressor rotor: (a) finite element model, and (b) tuned natural frequency curves. 


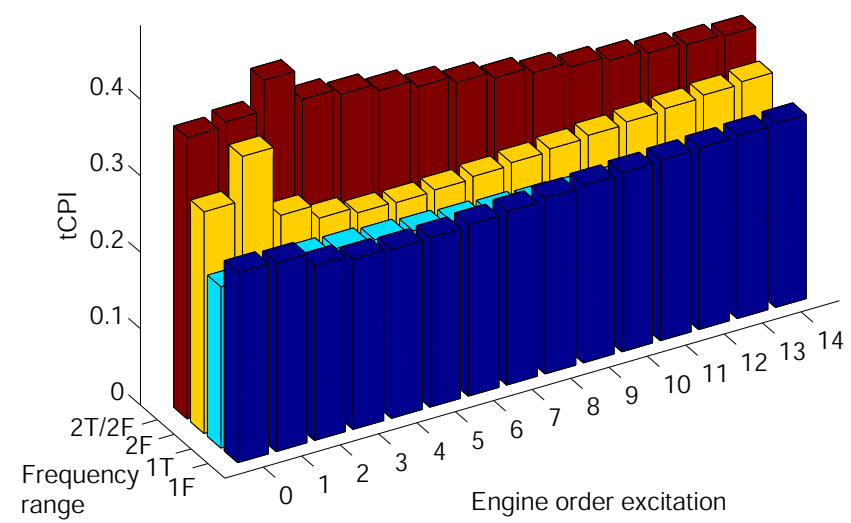

Figure 12. Disk-blade dynamic interactions, calculated by power flow analysis, for the tuned compressor rotor with nominal design configuration.

displayed in figure 12. The disk-blade interactions are largest for engine order 1 and 2 excitations of the $2 \mathrm{~F}$ and $2 \mathrm{~T} / 2 \mathrm{~F}$ blade mode families, respectively. Also, the $2 \mathrm{~T} / 2 \mathrm{~F}$ family features larger dynamic interactions than the $2 \mathrm{~F}$ family. Thus, for these operating conditions, large amplifications in blade forced responses are anticipated relative to other operating conditions. In order to verify this conjecture, Monte Carlo simulations on the SAI metric are performed, and figures 13 a) and (b) confirm this initial prediction; therefore, these two cases of engine order and blade mode family are now selected as operating conditions of interest.

Next, power flow analysis is employed to predict variations in mistuning sensitivity, when disk structural parameters are changed arbitrarily. In doing so, the stiffness and mass density of the disk are perturbed $\pm 10 \%$ from nominal values, in a manner similar to the academic bladed disk. In addition to varying the structural properties, the number of constrained nodes associated with the interface where the disk is attached to the rotor shaft, is also changed; for details, see figures 14(a) to (h). Although this procedure is somewhat simplistic in terms of industrial application, it can possibly represent the disk design process in practice. For each design configuration, the operating conditions are given as engine order 1 and 2 excitations for the $2 \mathrm{~F}$ and $2 \mathrm{~T} / 2 \mathrm{~F}$ blade mode families, as predicted from the case study

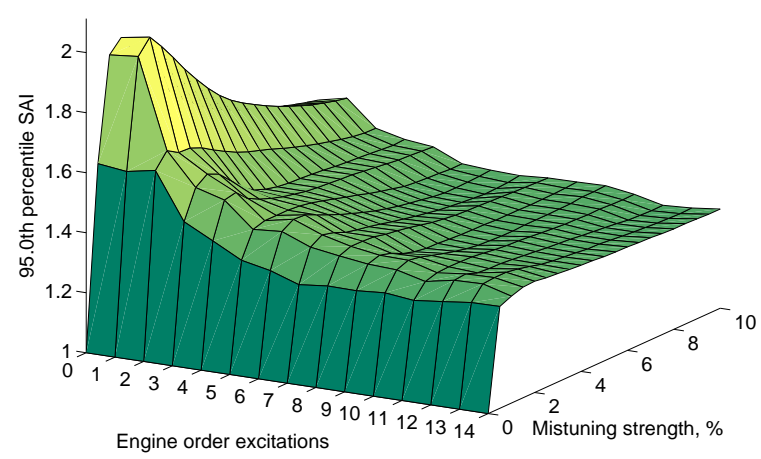

(a) $2 \mathrm{~F}$ veering region

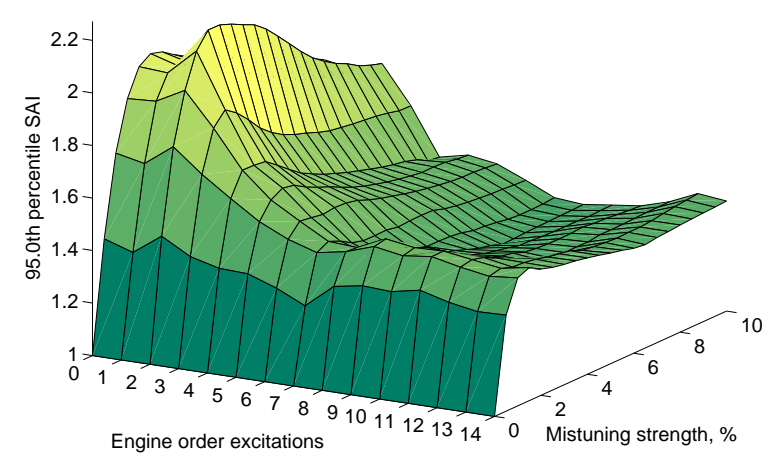

(b) $2 \mathrm{~T} / 2 \mathrm{~F}$ veering region

Figure 13. Monte Carlo simulations of the compressor rotor with nominal design, for different operating conditions. 


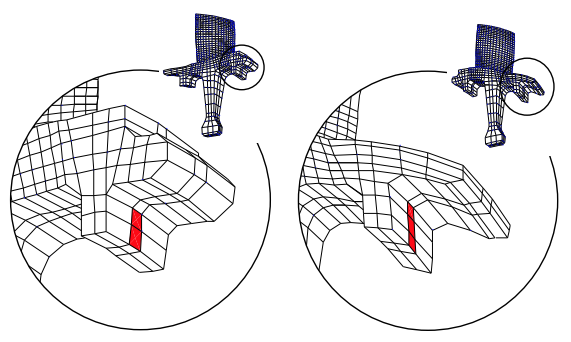

(a)

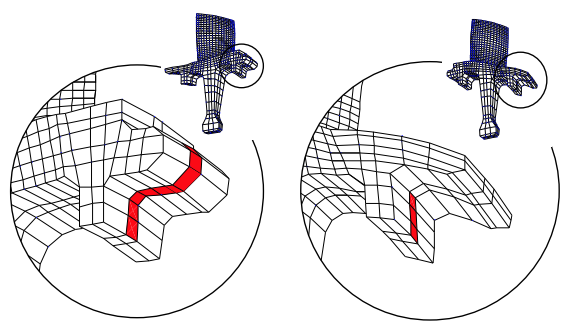

(c)

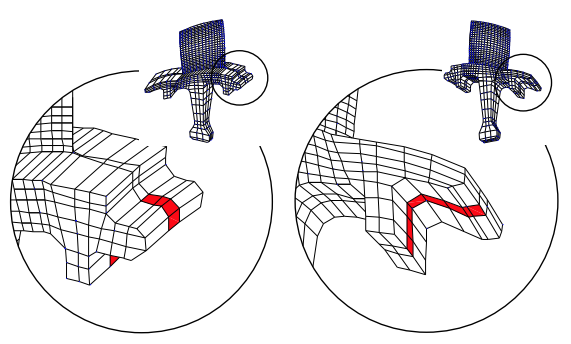

(e)

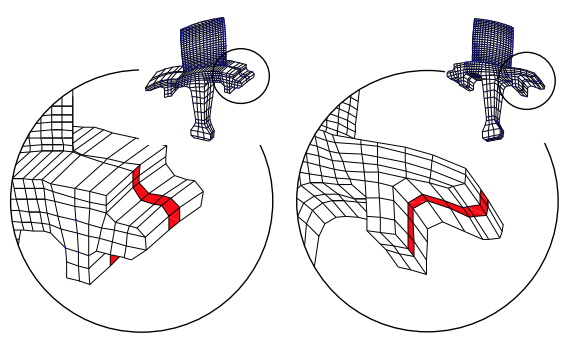

(g)

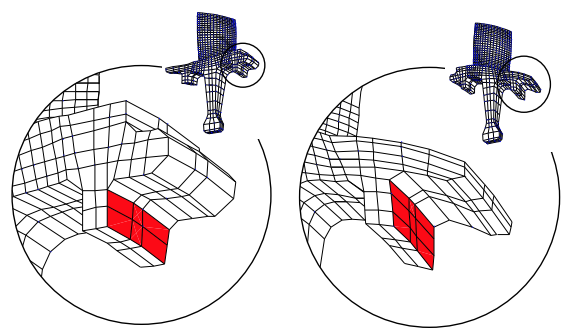

(b)

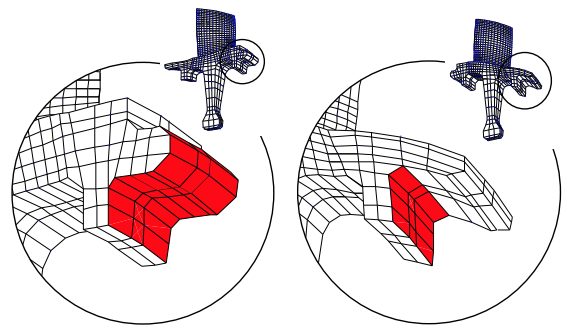

(d)

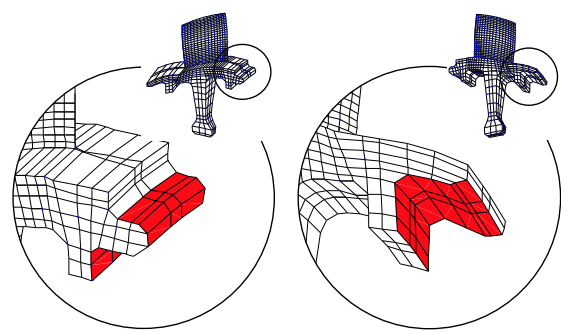

(f)

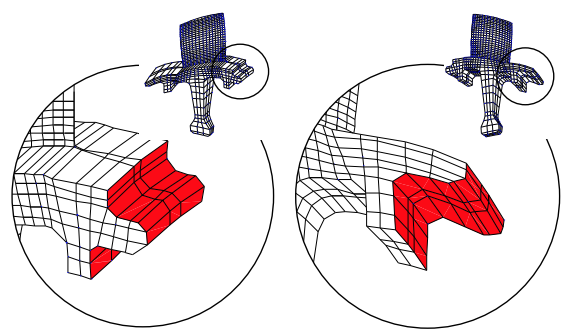

(h)

Figure 14. Variations in the disk fixture design, implemented by changing the boundary condition. The nodes in the darker area, corresponding to the disk surface in contact with rotor shaft, are fixed. The boundary condition change, depicted for the single sector, is then applied to the full bladed disk model. 
of the nominal design. Monte Carlo simulations of mistuned bladed disks are performed and the power flow is calculated for single tuned sectors; the analysis results are plotted against each other in figure 15. Observe that for all these arbitrary disk design changes, the power flow indicator (tCPI) successfully predicts the monotonic variations in blade stress amplification caused by mistuning.

\section{Case Study: Advanced Bladed Disk}

As a final case study, the power flow analysis is implemented for evaluating mistuning sensitivity of an advanced rotor stage design. The bladed disk features unique characteristics in terms of frequency veering phenomena. Thus, this numerical experiment can possibly be viewed as an extreme test of the proposed methodology.

Figure 16(a) shows the finite element model of a next-generation compressor stage, referred to herein as the advanced bladed disk. This 26-bladed blisk is made of a tungsten alloy, whose material properties are listed in table 4, and its inner hub is clamped to a custom-made

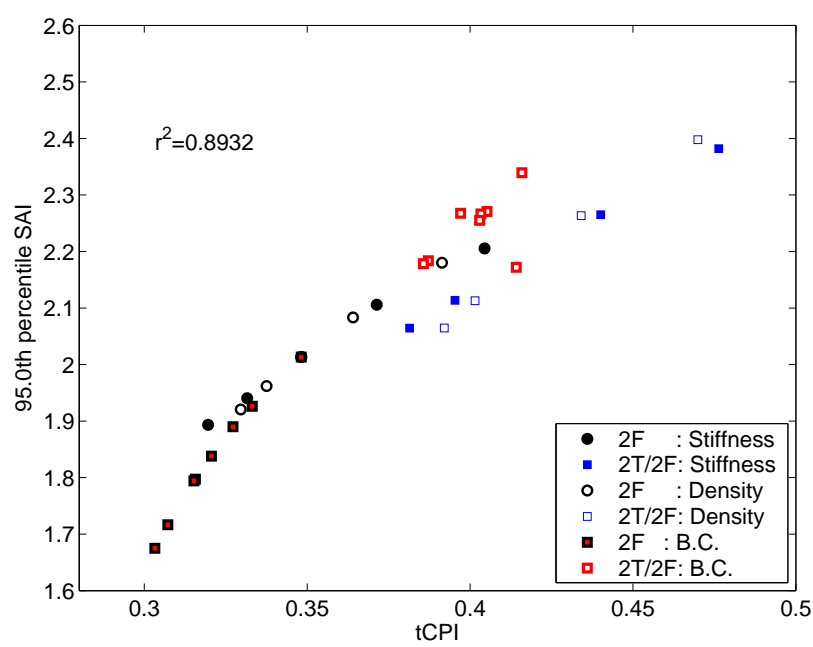

Figure 15. Assessments of blade mistuning effects for the compressor rotor for arbitrary disk design changes. The legend, "B.C.", denotes the cases for which the number of constrained nodes is changed. fixture. It is modeled with tetragonal pyramid solid elements with 10 nodes per element. The total number of DOF is 1,306,500 and the single sector model has 53,451 DOF.

At first, the tuned dynamic characteristics are investigated. Figure 16 b) shows the tuned free vibration natural frequencies versus the number of nodal diameters. The continuous veering plots are shown, and the various families of disk- and blade-dominated modes are also identified; for details see Ref. 12 There is a veering near nodal diameter

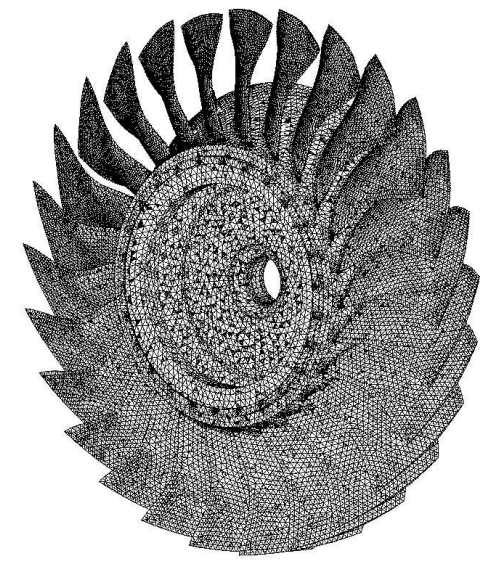

(a)

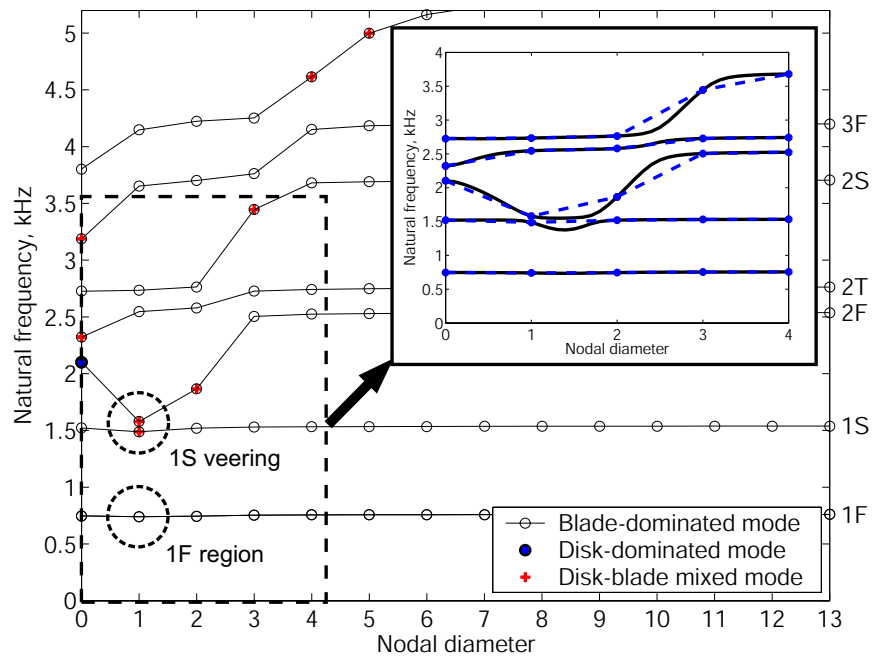

(b)

Figure 16. Advanced bladed disk assembly: (a) finite element model, and (b) tuned natural frequency curves. 
Table 4. Material properties of the advanced bladed disk.

\begin{tabular}{lcc} 
Material property & Value & Unit \\
\hline Modulus of Elasticity & 117.4 & $\mathrm{GPa}$ \\
Mass Density & 4653 & $\mathrm{~kg} / \mathrm{m}^{3}$ \\
Poisson's ratio & 0.310 & - \\
Structural damping & 0.006 & -
\end{tabular}

1 for the 1S blade mode family, as circled on the figure; however, the veering geometry shown in the magnified plot is somewhat uncommon. Moreover, the nodal diameter 1 mode of the $1 \mathrm{~F}$ family, also circled, has a slightly lower natural frequency relative to others. In additions, there are few disk-dominated modes in this range, since the large blades typically dominate the vibration of this structural system.

This bladed disk also possesses very unique dynamic characteristics when mistuned. Figures 17 (a) and (b) shows Monte Carlo simulation results for the above two frequency ranges. A unit external excitation is given to the nodes at the blade tip, perpendicularly to the blade surface. Observe that for the $1 \mathrm{~S}$ blade-mode family excitation, the blade response increases drastically for an engine order 1 excitation, with a stress amplification indicator of more than 3.5. Perhaps more surprisingly, for the $1 \mathrm{~F}$ blade-mode excitation, a very large amplification of more than 2.4 is also observed for engine order 1 excitation, although no curve veering is found near this operating condition. Thus, the usual veering analysis techniques, based on modeling modal dynamic interactions between adjacent modes, such as those in Refs. 11 and 12, may not work for this unusual operation condition.

Power flow analysis is now applied to the advanced bladed disk. For the nominal design, the tCPI values are computed for the $1 \mathrm{~F}$ and $1 \mathrm{~S}$ blade-mode family excitations. As observed in figure 18 , the power flow analysis clearly predict the considerable blade mistuning effects for engine order 1 excitation for both of these frequency ranges. Moreover, the disk-blade dynamic interactions are far stronger for the $1 \mathrm{~S}$ blade-modes family. This also does match with the Monte Carlo simulations of mistuned bladed disks reported in figure 17.

The power flow analysis can also be demonstrated to predict the trends of mistuning sensitivity caused by disk

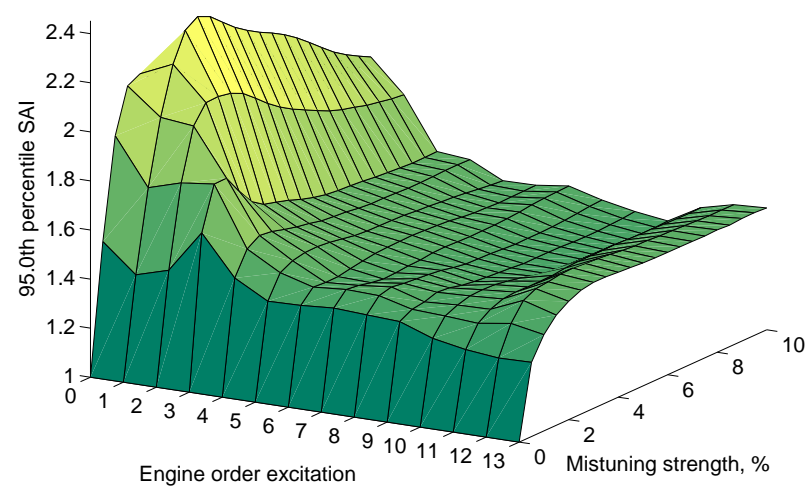

(a) $1 \mathrm{~F}$ frequency range

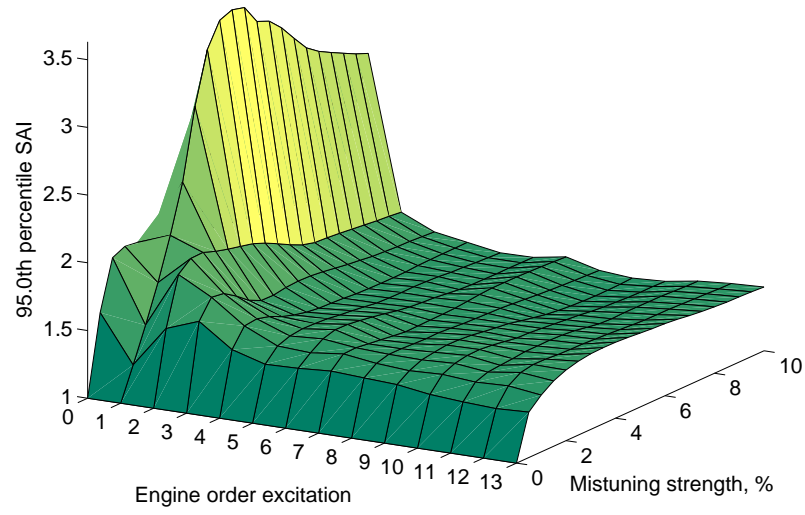

(b) $1 \mathrm{~S}$ frequency range

Figure 17. Monte Carlo simulations of the SAI values for the mistuned advanced bladed disks with nominal design configuration. 


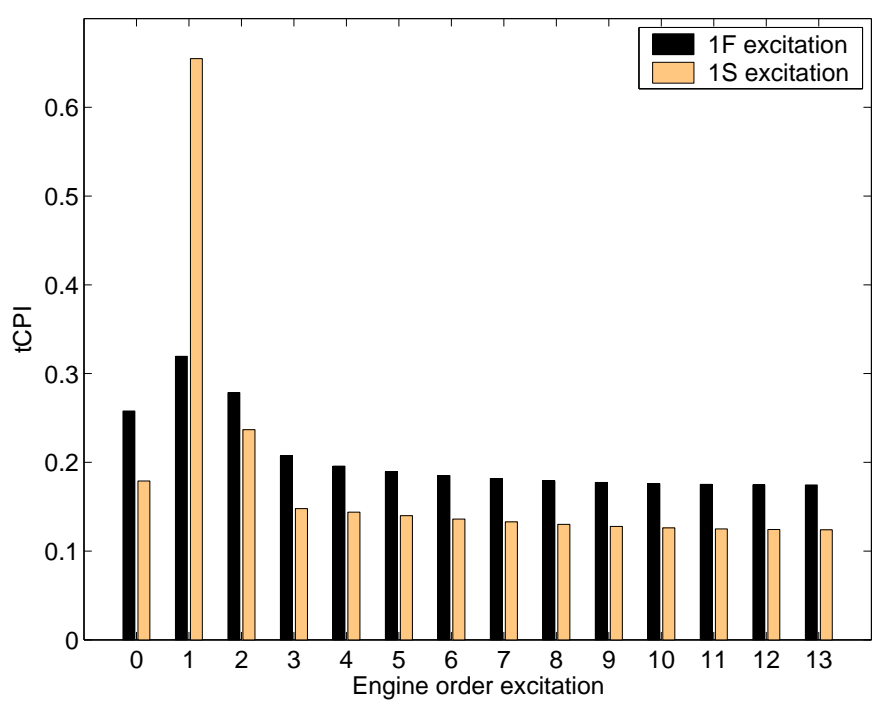

Figure 18. The tCPI values, obtained from power flow analysis, for the advanced bladed disk with nominal design configuration.

design changes. Similarly to the previous bladed disk models, the disk structural parameters (stiffness and mass density) are perturbed $\pm 10 \%$ from their nominal values. The operating condition selected is an engine order 1 excitation for both the $1 \mathrm{~F}$ and $1 \mathrm{~S}$ frequency ranges, based on the results for the nominal bladed disk. The tCPI and SAI values are calculated for the various disk design configurations, and plotted against each other in figure 19. It demonstrates that the coupling power flow indicator works well in capturing the dependence of the blade stress amplification due to mistuning with respect to the disk design changes.

Lastly, in order to validate the consistency of the power flow analysis, the numerical experiment results for the three bladed disk models are represented in a single plot. As observed in figure 20, the disk-blade dynamic interaction,

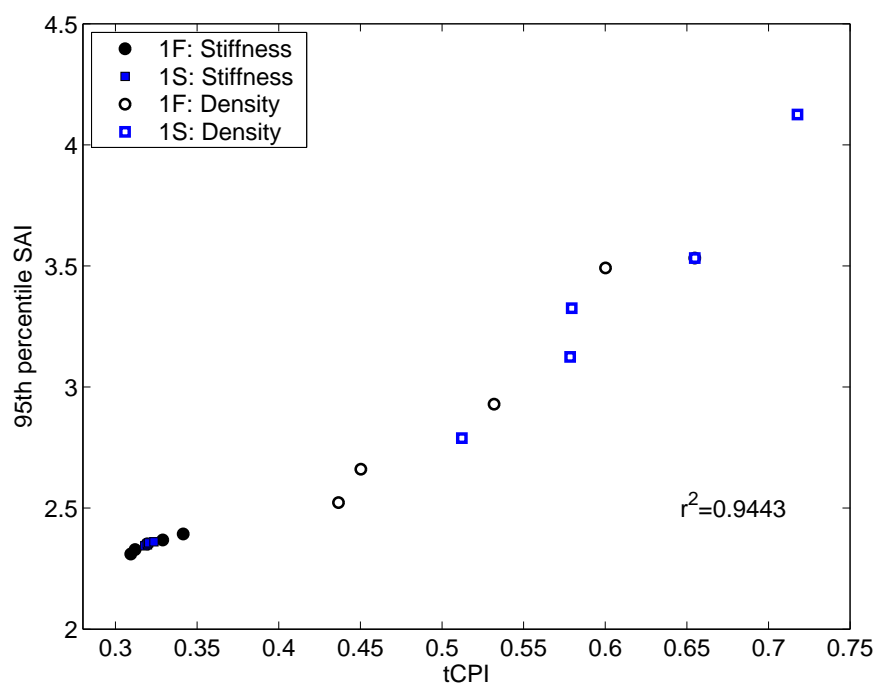

Figure 19. Assessment of blade mistuning effects for the advanced bladed disk, when changing disk structural parameters arbitrarily. 


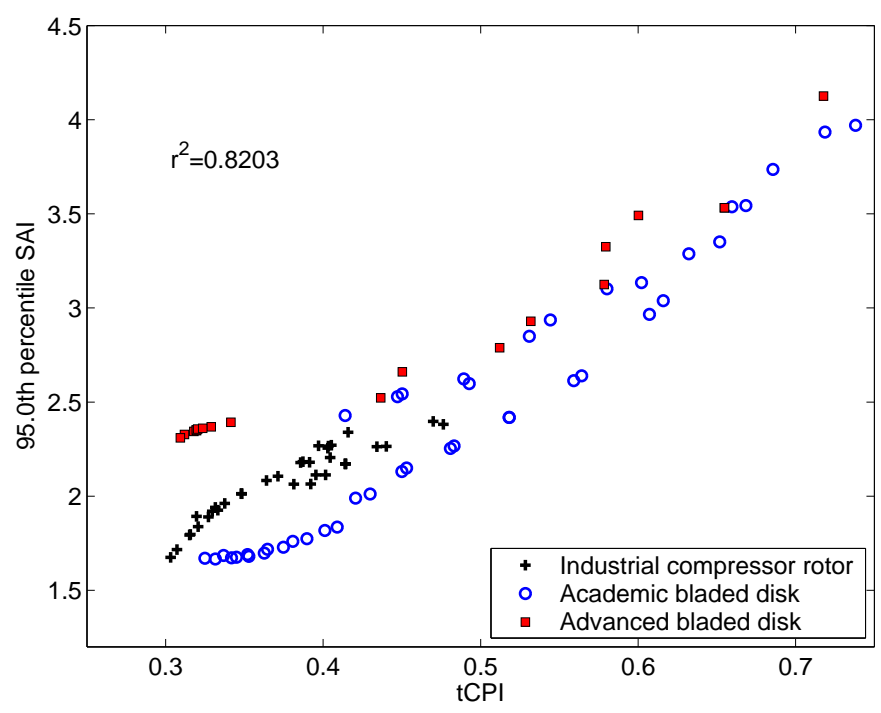

Figure 20. Prediction of blade stress amplifications due to mistuning for the three bladed disk models with arbitrary disk design changes.

predicted by the power flow analysis of a single tuned sector, is able to screen out dangerous bladed disk designs with respect to mistuning effects on blade stress increases. Hence, the initial evaluation of mistuning sensitivity, which is usually done via costly statistical methods such as Monte Carlo simulations, can now be performed very quickly, albeit approximately. Once dangerous operating conditions and designs have been identified by high tCPI values, complete Monte Carlo simulations can then be performed for these restricted cases.

\section{Conclusion}

An efficient method for the early assessment of bladed disk designs with respect to blade mistuning effects was presented. The power flow analysis developed provides an explanation as to why mistuned blade forced response increases relative to the tuned response when the disk and the blades feature strong dynamic interactions; namely, strong disk-blade coupling provides a sufficient amount of vibration within the disk, and the effect of blade mistuning is to reverse the energy flow from the disk to the blades, consequently causing a significant increase in blade forced response amplitudes. Disk-blade dynamic interactions are similar for tuned and mistuned systems, which enables one to estimate blade mistuning effects using the blade-to-disk coupling power flow for the tuned bladed disk. This was validated for lumped-parameter models and demonstrated for finite element models of industrial bladed disks.

The quick estimates of blade mistuning effects obtained from the power flow analysis of a tuned sector can be used to guide design modifications and produce optimal solutions of bladed disk designs that minimize mistuning effects on blade forced response. Then, more advanced and expensive analyses can be carried out for the finalized design, such as reduced-order modeling of the finite element model and the associated Monte Carlo simulations of blade forced response.

\section{Acknowledgments}

This work is supported by the GUIde Consortium on Blade Durability at Carnegie Mellon University. The authors gratefully acknowledge the NASA Glenn Research Center, which provided the finite element model of the advanced bladed disk. 


\section{References}

${ }^{1}$ Srinivasan, A. S., "Flutter and Resonant Vibration Characteristics of Engine Blades," ASME Journal of Engineering for Gas Turbines and Power, Vol. 119, 1997, pp. 742-775.

${ }^{2}$ Bendiksen, O. O., "Localization Phenomena in Structural Dynamics," Chaos, Solitions and Fractals, Vol. 11, 2000, pp. 1621-1660.

${ }^{3}$ Yang, M. T., and Griffin, J. H., "A Reduced-Order Model of Mistuning Using a Subset of Nominal System Modes," ASME Journal of Engineering for Gas Turbines and Power, Vol. 123, 2001, pp. 893-900.

${ }^{4}$ Bladh, R., Pierre, C., Castanier, M. P., and Kruse, M. J., "Dynamics Response Predictions for a Mistuned Industrial Turbomachinery Rotor Using Reduced-Order Modeling," ASME Journal of Engineering for Gas Turbines and Power, Vol. 124, 2002, pp. 311-324.

${ }^{5}$ Lim, S., Bladh, R., Castanier, M. P., and Pierre, C., "A Compact, Generalized Component Mode Mistuning Representation for Modeling Bladed Disk Vibrations," AIAA Paper 2003-1545, Proceedings of the 44th AIAA/ASME/ASCE/AMS Structures, Structural Dynamics, and Material Conference, Norfolk, VA, 2003.

${ }^{6}$ Cha, D., and Sinha, A., "Statistics of Response of a Mistuned Bladed Disk Assembly Subjected to White Noise and Narrow Band Excitation," Proceedings of International Gas Turbine and Aeroengine, Congress and Exhibition, Stockholm, Sweden, 1998.

${ }^{7}$ Whitehead, D. S., "The Maximum Factor by Which Forced Vibration of Blades Can Increase Due to Mistuning," ASME Journal of Engineering for Gas Turbines and Power, Vol. 120, 1998, pp. 115-119.

${ }^{8}$ Mignolet, M. P., Lin, C.-C., and LaBorde, B. H., "A Novel Limit Distribution for the Analysis of Randomly Mistuned Bladed Disks," ASME Journal of Engineering for Gas Turbines and Power, Vol. 123, 2001, pp. 388-394.

${ }^{9}$ Castanier, M. P., Pierre, C., and Bladh, R., "Investigation into Using Tuned Free Response Information to Predict Mistuned Forced Response of Bladed Disks," Proceedings of the 7th National Turbine Engine High Cycle Fatigue Conference, Palm Beach Gardens, FL, 2002.

${ }^{10}$ Hussein, M. I., Pierre, C, and Castanier, M. P., "Correlation of Tuned Free Vibration Characteristics with Mistuning Sensitivity for A Bladed Disk," AIAA Paper 2003-1544, Proceedings of the 44th AIAA/ASME/ASCE/AMS Structures, Structural Dynamics, and Material Conference, Norfolk, VA, 2003.

${ }^{11}$ Kenyon, J. A., and Griffin, J. H., "Mistuned Bladed Disk Forced Response with Frequency Veering," Proceedings of the 8th National Turbine Engine High Cycle Fatigue Conference, Monterey, CA, 2003.

${ }^{12}$ Baik, S., Castanier, M. P., and Pierre, C., "Mistuning Sensitivity Predictions of Bladed Disks Using Eigenvalue Curve Veerings," Proceedings of the 9th National Turbine Engine High Cycle Fatigue Conference, Pinehurst, NC, 2004.

${ }^{13}$ Ottarsson., G. S., and Pierre, C., "On the Effects of Interblade Coupling on the Statistics of Maximum Forced Response Amplitudes in Mistuned Bladed Disks," Proceedings of the 36th AIAA/ASME/ASCE/AHS Structures, Structural Dynamics, and Material Conferences, New Orleans, LA, 1995, pp. 3070-3078.

${ }^{14}$ Bladh, R., Castanier, M. P., and Pierre, C., "Component-Mode-Based Reduced Order Modeling Techniques for Mistuned Bladed Disks, Part II: Application,” ASME Journal of Engineering for Gas Turbines and Power, Vol. 123, 2001, pp. 100-108. 